\title{
Magnesium Alloys for Biomedical Applications
}

\author{
Petra Maier ${ }^{1, *(\mathbb{D})}$ and Norbert Hort ${ }^{2}$ \\ 1 Hochschule Stralsund-University of Applied Sciences Stralsund, 18435 Stralsund, Germany \\ 2 Helmholtz-Zentrum Geesthacht-Magnesium Innovation Centre, 21502 Geesthacht, Germany; \\ norbert.hort@hzg.de \\ * Correspondence: petra.maier@hochschule-stralsund.de; Tel.: +49-3831-456419
}

Received: 28 September 2020; Accepted: 3 October 2020; Published: 5 October 2020

\section{Introduction and Scope}

Interest in magnesium alloys as biodegradable metals has strongly grown over the last decades. Magnesium alloys are of interest mainly because of their biocompatibility, moderate corrosion rate, and appropriate mechanical properties when the right alloying system and process parameters are used. Potential applications of magnesium alloys are represented by structural material for orthopedics and temporary cardiovascular devices. These implants are absorbed by the body after completing their temporary functions, like mechanical support, scaffolding, and bonding to living tissue. The focus in the development of magnesium alloys is on alloy design, alloy fatigue and stress corrosion properties, deformability, moderate corrosion rate, and uniform corrosion morphology. The community is highly discussing the relation between magnesium alloys' in vitro and in vivo properties, which would help to reduce animal testing and support simulations to pre-select alloys. Mechanical properties are usually described by tensile tests and hardness tests. Furthermore, fatigue and stress corrosion data are needed to get a full picture of stability over degradation time. Magnesium alloys are known for pitting corrosion. Since corrosion pits cause increased stress intensity under mechanical loading and early failure of implants, special attention should be paid to a uniform corrosion morphology. The aim of this Special Issue on biodegradable magnesium is to explore and introduce new alloys to overcome the current limitations of magnesium applications.

\section{Contributions}

Four research articles and one review paper have been published in this Special Issue of Metals. The subjects cover studies on corrosion behavior and its characterization in in-vitro environments [1-3] and in vivo studies on facial reconstruction [4,5]. Mainly Al-free alloys, Mg-Gd [1], Mg-Zn-Ca [2], $\mathrm{Mg}-\mathrm{Dy}-\mathrm{Nd}$ [3], and $\mathrm{Mg}-\mathrm{Zn}-\mathrm{Zr}$ [5] and its corrosion morphology are the focus of papers in this Special Issue.

The first article by Gawlik et al. [1] on "Acetic Acid Etching of Mg-xGd Alloys" investigates a suitable etching method, here acetic acid (HAc) etching, to remove surface contamination and to understand the influence of etching on surface morphology. Extruded $\mathrm{Mg}-\mathrm{Gd}$ discs were etched with HAc solution in different volumes and times, to remove contamination from the surface. Surface characterization was done by various analyzing techniques and the microstructure near the etched surface was also looked at. It has been found that Gd-rich phases and twins promote the formation of pits, but increase in temperature of the exothermic reaction has the highest impact on pit formation. Short etching leads to a removal of a few $\mu \mathrm{m}$ of material from initial smooth surfaces, resulting in a plain surface morphology and the removal of surface Fe contamination. Rougher surfaces need longer etching times. Smooth surfaces can reduce scattering of degradation rates.

In order to imitate the real service environment, surface morphologies and mechanical properties of amorphous $\mathrm{Mg}-\mathrm{Zn}-\mathrm{Ca}$ alloys were studied by Li et al. [2] under different chemistry mechanics 
interactive environments. Compared to crystalline alloys, the material in its amorphous condition exhibits higher compressive strength and corrosion resistance due to their uniform composition contribution and almost crystal-defect-free microstructure. The authors found that $\mathrm{Ca} / \mathrm{Mg}$ phosphates and cracks formed on the surface, spreading and deepening gradually with increasing corrosion time and pressure. A brittle fracture was explored by fan-shaped patterns, clear cracks, and shear bands. The good compressive strength of amorphous $\mathrm{Mg}-\mathrm{Zn}-\mathrm{Ca}$ could avoid material failure: vein-like patterns in some areas support the appearance of some local plastic deformation. The paper is of referential significance for designing related experiments.

An extruded Mg-Dy-Nd alloy during stress corrosion with C-ring compression tests in Ringer solution has been examined in [3]. Microstructural changes during deformation, initial hardness, and strength are used to discuss stress corrosion behavior. The ternary alloy $\mathrm{Mg}-\mathrm{Dy}-\mathrm{Nd}$ has the highest hardness and strength but lacks ductility. The alloys twin during plastic deformation. None of the alloys failed by stress corrosion cracking at moderate deformation, but corrosion pits formed to various extents. The pits were found to be of elliptical shape, and they overlap during ongoing corrosion. Crack propagation in C-ring samples, which were subjected to compressive loading until fracture, was influenced by the twinned microstructure. The $\mathrm{Mg} 1 \mathrm{Nd}$ alloy even twinned the most, showed ductile behavior, and higher corrosion resistance. The Dy-containing alloys indicate features of hydrogen-assisted fracture. Mg10Dy showed a higher fracture toughness than Mg10Dy1Nd, mainly because of its higher ductility.

"The Potential of Magnesium Based Materials in Mandibular Reconstruction" was reviewed by Prasadh et al. [4]. They gave an overview of permanent biomaterials and put them in contrast to bioresorbable Mg-based materials. Permanent biomaterials such as Ti6Al4V alloy, 316L stainless steel, and Co-based alloys currently used in mandibular reconstruction often result in stress shielding effects due to a mismatch in the Young's modulus values between the bone and the implant, resulting in implant loosening and allergic responses. The authors proposed Mg-based materials as revolutionary biomaterials that have transformed the nature of metallic biomaterials from bioinert to bioactive and multi-bio functional Mg-alloys that exhibit favorable mechanical properties, adequate biocompatibility, and degradability. The paper reviews the recent progress in developing Mg-based materials for mandibular reconstruction, taking the mechanical properties, corrosion characteristics, and in vivo performance in correlation with the biomechanics of the mandible and types of mandibular defects into account. In general, surgical components like screws, plates, wires, and other temporary devices are possible applications.

Byun et al. [5] evaluated the mechanical strength and biodegradation of a ZK60 plate coated with poly(l-lactic)-acid polymer (PLLA) in a LeFort I osteotomy canine model for maxillofacial applications based on dogs. The presence of wound dehiscence, plate exposure, gas formation, inflammation, pus formation, occlusion, food intake, and fistula formation were observed and discussed. After 12 weeks X-ray microcomputed tomography $(\mu \mathrm{CT})$ was conducted and plates could not be found anymore. Plate exposure, gas formation, and external fistula could not be seen, and the occlusion remained stable. However, wound dehiscence did not heal for 12 weeks. A few screw bodies fixed in the bone remained, but screw heads were completely absorbed: here an optimization of the absorption rate by the PLLA coating is aimed. Rapid degradation of PLLA-coated ZK60 is based on the formation of microcracks during bending, needed for precise adaptation in fixation surgery: improvements to the plate system using other surface coating methods or alternative $\mathrm{Mg}$ alloys are required.

\section{Conclusions and Outlook}

The present Special Issue of Metals provided a comprehensive insight into the current state of research on developing magnesium alloys as biodegradable implant materials. Alloy development needs to address degradation behavior in vivo in relation to in vitro and mechanical properties as well as preparation methods to characterize corrosion morphology. We are confident that this Special Issue will be valuable for future developments and discussions. 
As guest editors of this Special Issue, we hope that the presented papers will be useful to researchers, designers, and practitioners developing and working towards the application of magnesium alloys as implants. We would like to express our gratitude to all authors for their contributions as well as to anonymous reviewers for their efforts in ensuring a high-quality publication. Special thanks also to editors of Metals for their help, and to the editorial assistants of Metals for their valuable engagement and support during the preparation of this Special Issue.

Conflicts of Interest: The authors declare no conflict of interest.

\section{References}

1. Gawlik, M.M.; Wiese, B.; Welle, A.; González, J.; Desharnais, V.; Harmuth, J.; Ebel, T.; Willumeit-Römer, R. Acetic Acid Etching of Mg-xGd Alloys. Metals 2019, 9, 117. [CrossRef]

2. Li, Y.; Liang, Z.; Yang, Y.; Zhao, W.; Wang, Y.; Yu, H.; Qin, C.; Wang, Z. Surface Morphologies and Mechanical Properties of Mg-Zn-Ca Amorphous Alloys under Chemistry-Mechanics Interactive Environments. Metals 2019, 9, 327. [CrossRef]

3. Maier, P.; Clausius, B.; Wicke, J.; Hort, N. Characterization of an Extruded Mg-Dy-Nd Alloy during Stress Corrosion with C-Ring Tests. Metals 2020, 10, 584. [CrossRef]

4. Prasadh, S.; Ratheesh, V.; Manakari, V.; Parande, G.; Gupta, M.; Wong, R. The Potential of Magnesium Based Materials in Mandibular Reconstruction. Metals 2019, 9, 302. [CrossRef]

5. Byun, S.-H.; Lim, H.-K.; Lee, S.-M.; Kim, H.-E.; Kim, S.-M.; Lee, J.-H. Biodegradable Magnesium Alloy (ZK60) with a Poly(l-lactic)-Acid Polymer Coating for Maxillofacial Surgery. Metals 2020, 10, 724. [CrossRef]

(C) 2020 by the authors. Licensee MDPI, Basel, Switzerland. This article is an open access article distributed under the terms and conditions of the Creative Commons Attribution (CC BY) license (http://creativecommons.org/licenses/by/4.0/). 